\title{
Observational constraints on interacting dark matter model without dark energy (Research Note)
}

\begin{abstract}
S. Cao, Z.-H. Zhu, and N. Liang
Department of Astronomy, Beijing Normal University, Beijing 100875, PR China

e-mail: [zhuzh,liangn]@bnu.edu.cn

Received 25 November 2010 / Accepted 2 March 2011

\section{ABSTRACT}

Aims. The interacting dark matter (IDM) scenario allows for the acceleration of the Universe without dark energy.

Methods. We constrain the IDM model by using the newly revised observational data including $H(z)$ data and Union2 SNe Ia via the Markov chain Monte Carlo method.

Results. When mimicking the $\Lambda \mathrm{CDM}$ model, we obtain a more stringent upper limit to the effective annihilation term at $\kappa C_{1} \approx$ $10^{-3.4} \mathrm{Gyr}^{-1}$, and a tighter lower limit to the relevant mass of dark matter particles at $M_{x} \approx 10^{-8.6} \mathrm{Gev}$. When mimicking the $w \mathrm{CDM}$ model, we find that the effective equation of state of IDM is consistent with the concordance $\Lambda$ CDM model and appears to be most consistent with the effective phantom model with a constant EoS for which $w<-1$.
\end{abstract}

Key words. dark matter - cosmology: observations

\section{Introduction}

Recent observations of type Ia sPerlmutterupernovae (SNe Ia, Riess et al. 1998; Perlmutter et al. 1999) have predicted that our present universe is passing through an accelerated phase of expansion proceeded by a period of deceleration. A new type of matter with negative pressure, which is popularly known as dark energy, has been proposed to explain the present phase of acceleration. The most simple dark energy candidate, the cosmological constant $(\Lambda \mathrm{CDM}$ model), though known to be consistent with various observations such as $\mathrm{SNe}$ Ia, the galaxy cluster gas mass fraction data (Wilson et al. 2006; Davis et al. 2007; Allen et al. 2008), and the CMB temperature and polarization anisotropies (Jassal et al. 2010), is always affected by the coincidence problem. Until now, many other dark energy models have been brought forward to explain this comic acceleration such as the scalar fields with a dynamical equation of state (e.g., quintessence, Peebles \& Ratra 1988a,b; Caldwell et al. 1998; phantom, Caldwell 2002; k-essence, Armendariz-Picon et al. 2001; quintom, Feng et al. 2005; Guo et al. 2005; Liang et al. 2009), the Chaplygin gas (Kamenshchik et al. 2001; Bento et al. 2002), holographic dark energy (Cohen 1999; Li 2004), and so on. Many alternatives to dark energy in which gravity is modified have been proposed as a possible explanation of the acceleration (e.g., the braneworld models, Dvali et al. 2000; Zhu \& Alcaniz 2005; the Cardassian expansion model, Freese \& Lewis 2002; Zhu \& Fujimoto 2002), as well as the $f(R)$ theory (Capozziello \& Fang 2002; Carroll et al. 2004) and the $f(T)$ theory (Bengochea \& Ferraro 2009; Wu \& Yu 2010).

It has been shown that the dark matter self-interactions could provide the accelerated expansion of the Universe without any dark energy component (Zimdahl et al. 2001; Balakin et al. 2003). In the framework of the Boltzmann formalism, if there is a disequilibrium between the dark matter particle creation and annihilation processes, an effective source term with negative pressure could be created. Basilakos \& Plionis (2009) investigated the circumstances under which the analytical solution space within the framework of the interacting dark matter (IDM) scenario allows for a late accelerated phase of the Universe, and find that the effective annihilation term of the simplest IDM model is quite small by using the nine observational $H(z)$ data points of Simon et al. (2005). The gravitational matter creation model that is fully dominated by cold dark matter (Lima et al. 2008; Basilakos \& Lima 2010) is mathematically equivalent to one case of the IDM models (Basilakos \& Plionis 2009).

In this paper, we use the newly revised $H(z)$ data (Stern et al. 2010; Gaztañaga et al. 2009) and the Union2 set of 557 SNe Ia (Amanullah et al. 2010) to constrain the relevant IDM models (Basilakos \& Plionis 2009) by using the Markov chain Monte Carlo (MCMC) method. This paper is organized as follows. In Sect. 2, we briefly indicate the basic equations of the IDM models. Observational data including $H(z)$ and SNe Ia are given in Sect. 3. In Sect. 4, MCMC constraint results from different combined data sets are illustrated. Finally, we summarize our main conclusions in Sect. 5.

\section{The basic equations of the IDM models}

We assume that the IDM density obeys the collisional Boltzmann equation (Kolb \& Turner 1990)

$\dot{\rho}+3 H \rho+\kappa \rho^{2}-\Psi=0$,

where $\Psi$ is the rate of creation of DM particle pairs and the annihilation parameter $\kappa=\langle\sigma u\rangle / M_{x}$ (where $M_{x}$ is the mass of the DM particle, $\sigma$ is the cross-section for annihilation, and $u$ is the mean particle velocity). Compared to the usual fluid equation, the effective pressure term is

$p_{\text {eff }}=\left(\kappa \rho^{2}-\Psi\right) / 3 H$. 
When the IDM particle creation term is larger than the annihilation term $\left(\kappa \rho^{2}-\Psi<0\right)$, IDM may serve as a negative pressure source in the global dynamics of the Universe (Zimdahl et al. 2001; Balakin et al. 2003). Basilakos \& Plionis (2009) phenomenologically identified two functional forms for which the previous Boltzmann equation can be solved analytically, only one of which is of interest since it indicates the dependence of the scale factor on a " $\propto a^{-3}$ ". We refer to Appendix B in Basilakos \& Plionis (2009) for details. We assume that

$\Psi(a)=a H(a) R(a)=C_{1}(n+3) a^{n} H(a)+\kappa C_{1}^{2} a^{2 n}$.

And the total energy density is

$\rho(a)=C_{1} a^{n}+a^{-3} \frac{F(a)}{\left[C_{2}-\int_{1}^{a} x^{-3} f(x) F(x) \mathrm{d} x\right]}$,

where $n, C_{1}$, and $C_{2}$ are the corresponding constants of the problem $\left(\kappa C_{1}\right.$ in the unit of $\left.\mathrm{Gyr}^{-1}\right)$, and the kernel function $F(a)=\exp \left[-2 \kappa C_{1} \int_{1}^{a} x^{n-1} / H(x) \mathrm{d} x\right]$. The first term on the right side of Eq. (4) obviously corresponds to the residual matter creation that results from the possible disequilibrium between the IDM particle creation and annihilation processes, while the second term can be viewed as the energy density of the self IDM particles that are dominated by the annihilation process.

\subsection{Model 1: mimicking the $\Lambda C D M$ model}

If $n=0$, the global density evolution can be transformed as

$\rho(a)=C_{1}+a^{-3} \frac{\mathrm{e}^{-2 \kappa C_{1}\left(t-t_{0}\right)}}{\left[C_{2}-\kappa Z(t)\right]}$,

where $Z(t)=\int_{t_{0}}^{t} a^{-3} \mathrm{e}^{-2 \kappa C_{1}\left(t-t_{0}\right)}$ (Basilakos \& Plionis 2009). At the present epoch, the density evolves according to $\rho(a) \simeq C_{1}+$ $a^{-3} / C_{2}$, which is approximately equivalent to the corresponding evolution in the $\Lambda$ CDM model, in which the $C_{1}$ term resembles the cosmological constant term $\left(\rho_{\Lambda}\right)$ and the $1 / C_{2}$ term resembles the density of matter $\left(\rho_{\mathrm{m}}\right)$. The Hubble parameter can be written as (Basilakos \& Plionis 2009)

$\left(\frac{H}{H_{0}}\right)^{2}=\Omega_{1,0}+\Omega_{2,0} a^{-3} \frac{\mathrm{e}^{-2 \kappa C_{1}\left(t-t_{0}\right)}}{1+\kappa C_{1}\left(\Omega_{2,0} / \Omega_{1,0}\right) Z(t)}$,

where $\Omega_{1,0}=8 \pi G C_{1} / 3 H_{0}^{2}$ and $\Omega_{2,0}=8 \pi G / 3 H_{0}^{2} C_{2}$, which relate to $\Omega_{\Lambda}$ and $\Omega_{\mathrm{m} 0}$ in the $\Lambda \mathrm{CDM}$ model, respectively. The mass of the DM particle can also be related to the range of $\kappa C_{1}$ (Basilakos \& Plionis 2009)

$M_{x}=\frac{1.205 \times 10^{-12}}{\kappa C_{1}} \frac{\langle\sigma u\rangle}{10^{-22}} \mathrm{GeV}$.

\subsection{Model 2: mimicking the wCDM model}

When the annihilation term is negligible $(\kappa=0)$ and the particle creation term dominates, it is straightforward to obtain the evolution of the total energy density (Basilakos \& Plionis 2009)

$\rho(a)=C_{1} a^{n}+\left(1 / C_{2}\right) a^{-3}$.

The conditions $n>-2$ implies that the IDM fluid has an inflection point, and the current model acts as the $w C D M$ model with a constant EoS with $w_{\text {IDM }}=-1-n / 3$, but with a totally different intrinsic nature. This situation is mathematically equivalent to the gravitational DM particle creation process within the context of non-equilibrium thermodynamics (Lima et al. 2008). The Hubble parameter is now given by

$\left(\frac{H}{H_{0}}\right)^{2}=\Omega_{1,0} a^{n}+\Omega_{2,0} a^{-3}$

where $\Omega_{2,0}=8 \pi G\left(C_{2}-C_{1}\right) / 3 H_{0}^{2}$ and $\Omega_{1,0}=8 \pi G C_{1} / 3 H_{0}^{2}$, respectively (Basilakos \& Plionis 2009).

\section{Observational data}

To constrain the relevant IDM models (Basilakos \& Plionis 2009), we use the newly revised $H(z)$ data (Stern et al. 2010; Gaztañaga et al. 2009) and the Union2 set of $557 \mathrm{SNe}$ Ia (Amanullah et al. 2010).

\subsection{The observational $H(z)$ data}

It is known that the Hubble parameter $H(z)$ depends on the differential age as a function of redshift $z$ in the form

$H(z)=-\frac{1}{1+z} \frac{\mathrm{d} z}{\mathrm{~d} t}$

which provides a direct measurement on $H(z)$ based on $\mathrm{d} z / \mathrm{d} t$. Jimenez et al. (2003) demonstrated the feasibility of this method by applying it to a $z \sim 0$ sample. Moreover, compared with other observational data, it is more rewarding to investigate the observational $H(z)$ data directly, because it can take the fine structure of $H(z)$ into consideration and then use the important information that this structure provides.

By using the differential ages of passively evolving galaxies determined from the Gemini Deep Deep Survey (GDDS) (Abraham et al. 2004) and archival data (Treu et al. 2001, 2002; Nolan et al. 2003a,b), Simon et al. (2005) determined nine values of the Hubble parameter $H(z)$ in the range $0 \leq z \leq 1.8$, which have been used to constrain the parameters of various dark energy models (Samushia \& Ratra 2006; Wei \& Zhang 2007; Wu \& Yu 2007a,b; Zhang \& Zhu 2007; Kurek \& Szydlowski 2007; Lazkoz \& Majerotto 2007; Sen \& Scherrer 2008; Yi \& Zhang 2007; Wan et al. 2007; Xu et al. 2008; Zhai et al. 2010). The $H(z)$ data at 11 different redshifts were determined from the differential ages of red-envelope galaxies (Stern et al. 2010), and two more Hubble parameter data points $H(z=0.24)=$ $79.69 \pm 4.61$ and $H(z=0.43)=86.45 \pm 5.96$ were obtained by Gaztañaga et al. (2009) from observations of BAO peaks (for a review of the observational $H(z)$ data, see Zhang et al. 2010). Studies using these newly $H(z)$ data for cosmological constraint include Gong et al. (2010), Liang et al. (2010a), Liang \& Zhu (2010), Cao \& Liang (2010), Ma \& Zhang (2011), and $\mathrm{Xu} \&$ Wang (2010c). We emphasize the use of the Hubble constant $H_{0}$ in our analysis. Many previous have determined its present value. Freedman et al. (2001) presented the final results of the Hubble Space Telescope (HST) key project that measured the Hubble constant $H_{0}=72 \pm 8 \mathrm{~km} \mathrm{~s}^{-1} \mathrm{Mpc}^{-1}$, Gott et al. (2001) and Chen et al. (2003) proposed that $H_{0}=$ $68 \mathrm{~km} \mathrm{~s}^{-1} \mathrm{Mpc}^{-1}$ was a more likely value, and Tammann et al. (2008) obtained $H_{0}=62.3 \pm 1.3 \mathrm{~km} \mathrm{~s}^{-1} \mathrm{Mpc}^{-1}$ from 28 independently calibrated Cepheids and the distant, Cepheidcalibrated SNe Ia. More recently, Riess et al. (2009) determined $H_{0}=74.2 \pm 3.6 \mathrm{~km} \mathrm{~s}^{-1} \mathrm{Mpc}^{-1}$ by combining the observations of 240 Galactic Cepheid variables using HST. In this work, we follow Basilakos \& Plionis (2009) and adopt 
Table 1. The observational $H(z)$ data (Stern et al. 2010; Gaztañaga et al. 2009; Freedman et al. 2001).

\begin{tabular}{c|llllllllllllll}
\hline \hline$z$ & 0 & 0.1 & 0.17 & 0.24 & 0.27 & 0.4 & 0.43 & 0.48 & 0.88 & 0.9 & 1.3 & 1.43 & 1.53 & 1.75 \\
\hline$H(z)$ & 72 & 69 & 83 & 79.69 & 77 & 95 & 86.45 & 97 & 90 & 117 & 168 & 177 & 140 & 202 \\
\hline $1 \sigma$ uncertainty & \pm 8 & \pm 12 & \pm 8 & \pm 4.61 & \pm 14 & \pm 17 & \pm 5.96 & \pm 60 & \pm 40 & \pm 23 & \pm 17 & \pm 18 & \pm 14 & \pm 40 \\
\hline
\end{tabular}

$H_{0}=72 \pm 8 \mathrm{~km} \mathrm{~s}^{-1} \mathrm{Mpc}^{-1}$. The observational $H(z)$ data are given in Table 1 . In this case, $\chi^{2}$ can be defined as

$\chi_{H}^{2}=\sum_{i}^{14} \frac{\left(H-H_{\mathrm{obs}}\right)^{2}}{\sigma_{H}^{2}}$

\subsection{The observational SNe la data}

$\mathrm{SNe}$ Ia have long been used as "standard candles". It is commonly believed that measuring both their redshifts and apparent peak fluxes gives a direct measurement of their luminosity distances, thus SNe Ia provide the strongest constraints on the cosmological parameters (Riess et al. 2004, 2007; Astier et al. 2006; Davis et al. 2007; Wood-Vasey et al. 2007; Kowalski et al. 2008; Hicken et al. 2009; Chen et al. 2010). The present analysis uses the Union2 (557 sample) data set of the Supernova Cosmology project covering a redshift range $0.015 \leq z \leq 1.4$ (Amanullah et al. 2010), which was used to constrain cosmological models in Wei (2010), Liang et al. (2010a,b), Liang et al. (2011), Xu \& Wang (2010a,b), and Wang et al. (2010).

In the calculation of the likelihood from $\mathrm{SNe} I a$, we marginalize the nuisance parameter by minimizing (Di Pietro \& Claeskens 2003),

$\chi_{\mathrm{SNe}}^{2}=A-\frac{B^{2}}{C}+\ln \left(\frac{C}{2 \pi}\right)$,

where $A=\sum_{i}^{557}\left(\mu^{\text {data }}-\mu^{\text {th }}\right)^{2} / \sigma_{i}^{2}, B=\sum_{i}^{557}\left(\mu^{\text {data }}-\mu^{\text {th }}\right) / \sigma_{i}^{2}, C=$ $\sum_{i}^{557} 1 / \sigma_{i}^{2}$, and the distance modulus is $\mu=5 \log \left(d_{L} / \mathrm{Mpc}\right)+25$, with the $1 \sigma$ uncertainty $\sigma_{i}$ from the observations of SNe Ia and the luminosity distance $d_{L}$ as a function of redshift $z$,

$d_{L}=(1+z) \int_{0}^{z} \frac{c \mathrm{~d} z^{\prime}}{H\left(z^{\prime}\right)}$

\section{Constraint results}

We define the total likelihood to be the product of the separate likelihoods of the two cosmological probes, in other words,

$\chi_{\text {total }}^{2}=\chi_{H}^{2}+\chi_{\text {SNe }}^{2}$.

To determine the best-fit cosmological parameters, we use a $\chi^{2}$ minimization and the $68.3 \%$ and $95.4 \%$ confidence levels are defined by the conventional two-parameters $\chi^{2}$ levels 2.30 and 6.17 , respectively. We perform a global fitting to determine the cosmological parameters using the Markov chain Monte Carlo (MCMC) method and our MCMC code is based on the publicly available package COSMOMC (Lewis \& Bridle 2002).

\subsection{Model 1: mimicking the $\Lambda C D M$ model}

In this model, there are two free parameters: $\Omega_{2,0}$ and $\kappa C_{1}$ (or $M_{x}$ ) for a flat background $\left(\Omega_{1,0}+\Omega_{2,0}=1\right)$. In this paper, we follow Basilakos \& Plionis (2009) and adopt $t_{0}=1 / H_{0}=$ 13.6 Gyr (roughly the age of the universe in the corresponding
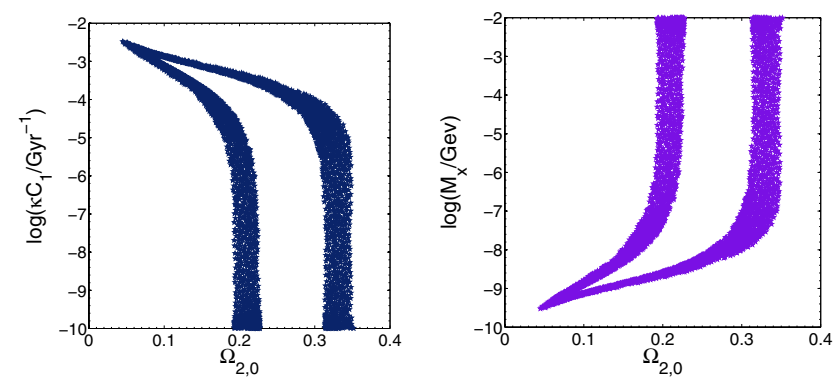

Fig. 1. The likelihood contours at the $68.3 \%$ and $95.4 \%$ confidence levels in the $\Omega_{2,0}-\kappa C_{1}$ and $\Omega_{2,0}-M_{x}$ planes provided by fitting model 1 to the $H(z)$ data.
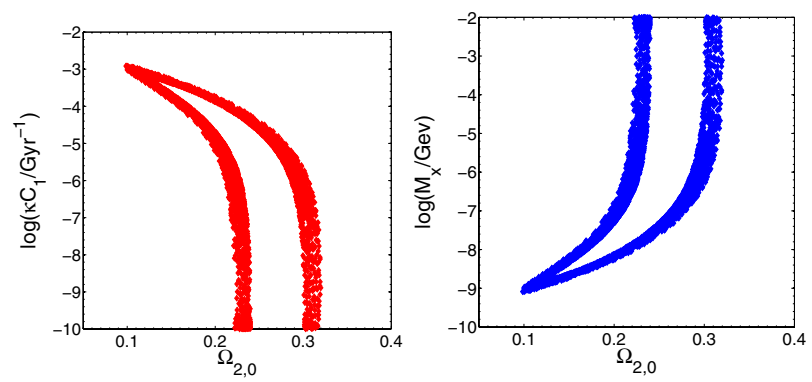

Fig. 2. The likelihood contours at the $68.3 \%$ and $95.4 \%$ confidence levels in the $\Omega_{2,0}-\kappa C_{1}$ and $\Omega_{2,0}-M_{x}$ planes provided by fitting model 1 to the $\mathrm{SNe}$ Ia data.
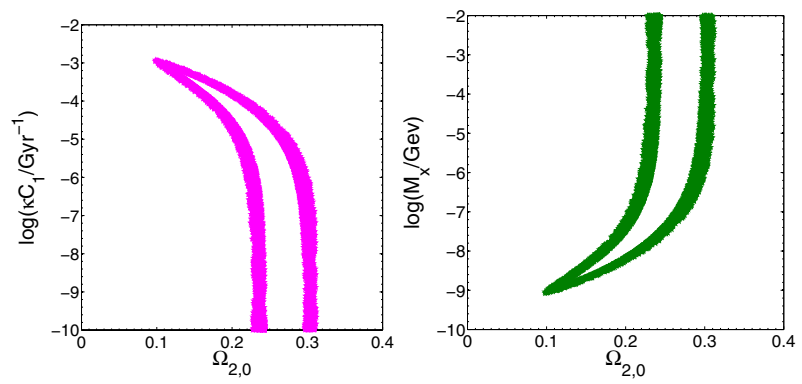

Fig. 3. The likelihood contours at the $68.3 \%$ and $95.4 \%$ confidence levels in the $\Omega_{2,0}-\kappa C_{1}$ and $\Omega_{2,0}-M_{x}$ planes provided by fitting model 1 to the $H(z)+\mathrm{SNe}$ data.

$\Lambda \mathrm{CDM}$ cosmology). The constraint results from different data combinations are shown in Figs. 1-3 and summarized in Table 2.

In Fig. 1, we show the results from the $H(z)$ data. The best-fit solution corresponds to fit $\Omega_{2,0}=0.270_{-0.044}^{+0.044}$ and $\log \left(\kappa C_{1} \cdot \mathrm{Gyr}\right) \approx-6.85$ with an upper limit $\log \left(\kappa C_{1} \cdot \mathrm{G}\right.$. Gyr $) \approx$ -3 . This effective annihilation term is obviously still unconstrained towards lower values. Correspondingly, the best-fit value of $M_{x}$ is $\log M_{x} / \mathrm{Gev} \approx-5.15$ with a relatively stringent lower limit of $\log M_{x} / \mathrm{Gev} \approx-9$. These results are consistent with previous work that uses fewer observational $H(z)$ data $\Omega_{2,0}=0.3_{-0.08}^{+0.05}$ and $\log \left(\kappa C_{1} \cdot\right.$ Gyr) $\approx-9.3$ (Basilakos \& Plionis 2009). In Fig. 2, we show the constraint results from SNe Ia with the best-fit model parameters $\Omega_{2,0}=0.271_{-0.031}^{+0.033}$ and $\log \left(\kappa C_{1} \cdot \mathrm{Gyr}\right) \approx-4.42$. A more stringent upper limit is obtained 
Table 2. Summarizing the results of parameter constraints from model 1 considered in this work.

\begin{tabular}{cccc}
\hline \hline Model 1 & $H(z)$ & $\mathrm{SNe}$ & $H(z)+\mathrm{SNe}$ \\
\hline$\chi_{\min }^{2}$ & 9.86 & 544.13 & 554.34 \\
$\Omega_{2,0}$ & $0.270_{-0.044}^{+0.044}$ & $0.271_{-0.031}^{+0.033}$ & $0.272_{-0.029}^{+0.028}$ \\
$\log \left(\kappa C_{1} \cdot \mathrm{Gyr}\right)$ & $-6.85(\leq-3)$ & $-4.42(\leq-3.5)$ & $-5.35(\leq-3.4)$ \\
$\log M_{x} / \mathrm{Gev}$ & $-5.15(\geq-9)$ & $-7.58(\geq-8.5)$ & $-6.65(\geq-8.6)$ \\
\hline
\end{tabular}

Notes. We adopt $t_{0}=1 / H_{0}=13.6 \mathrm{Gyr}$ in the computations.
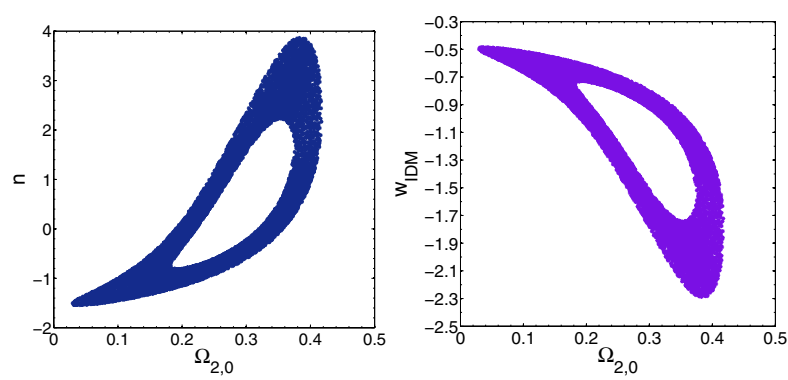

Fig. 4. The likelihood contours at the $68.3 \%$ and $95.4 \%$ confidence levels in the $\Omega_{2,0}-n$ and $\Omega_{2,0}-w_{\text {IDM }}$ planes provided by fitting model 2 to the $H(z)$ data.
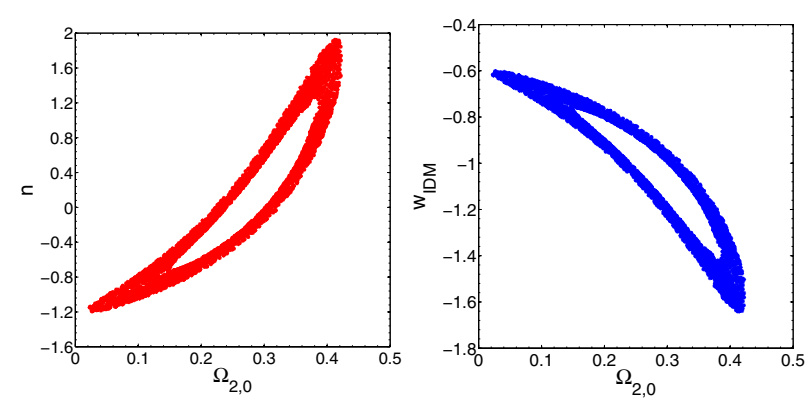

Fig. 5. The likelihood contours at the $68.3 \%$ and $95.4 \%$ confidence levels in the $\Omega_{2,0}-n$ and $\Omega_{2,0}-w_{\text {IDM }}$ planes provided by fitting model 2 to the $\mathrm{SNe}$ Ia data.

at around $\log \left(\kappa C_{1} \cdot \mathrm{Gyr}\right) \approx-3.5$. The best-fit DM particle mass is $\log M_{x} / \mathrm{Gev} \approx-7.58$ with a relatively stringent lower limit $\log M_{x} / \mathrm{Gev} \approx-8.5$. To obtain a tighter constraint on the model parameters, we combine the $H(z)$ and SNe Ia data, and the results are shown in Fig. 3. The best-fit model parameters are $\Omega_{2,0}=0.272_{-0.029}^{+0.028}$ and $\log \left(\kappa C_{1} \cdot \mathrm{Gyr}\right) \approx-5.35$ with a much more stringent upper $\operatorname{limit} \log \left(\kappa C_{1} \cdot \mathrm{Gyr}\right) \approx-3.4$. Moreover, the best-fit value of $M_{x}$ is $\log M_{x} / \mathrm{Gev} \approx-6.65$ with a relatively stringent lower limit of $\log M_{x} / \mathrm{Gev} \approx-8.6$ at $2 \sigma$. Since $M_{x}$ is unbound at small values, it is consistent with currently accepted lower bounds of $M_{x}(10 \mathrm{GeV})$ (see Cirelli et al. 2009, and references therein).

\subsection{Model 2: mimicking the wCDM model}

In this case, there are two free parameters: $\Omega_{2,0}$ and $n$ (or $w_{\text {IDM }}$ ). The constraint results from different data combinations are shown in Figs. 4-6 and summarized in Table 3.

For the $H(z)$ data and the results shown in Fig. 4, the best-fit parameter values are $\Omega_{2,0}=0.277_{-0.097}^{+0.098}$ and $n=$ $0.70_{-1.54}^{+1.54}$. For the EoS of IDM, the best-fit value is $w_{\text {IDM }}=$ $-1.23_{-0.51}^{+0.51}$. These results are consistent with the previous results of Basilakos \& Plionis (2009) by using fewer observational
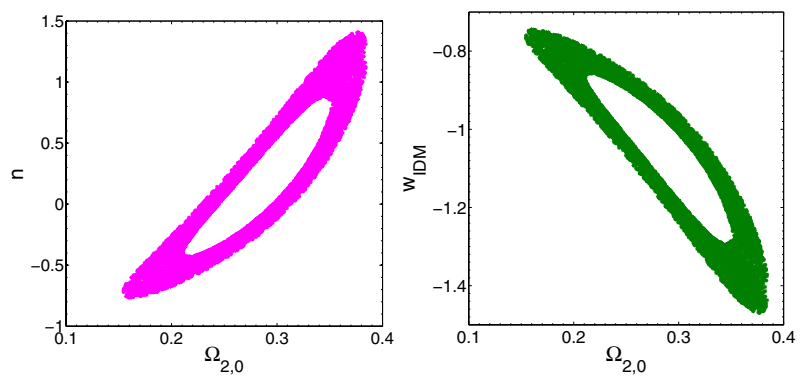

Fig. 6. The likelihood contours at the $68.3 \%$ and $95.4 \%$ confidence levels in the $\Omega_{2,0}-n$ and $\Omega_{2,0}-w_{\text {IDM }}$ planes provided by fitting model 2 to the $H(z)+\mathrm{SNe}$ data.

Table 3. Summarizing the results of constraint on parameters from model 2.

\begin{tabular}{cccc}
\hline \hline Model 2 & $H(z)$ & $\mathrm{SNe}$ & $H(z)+\mathrm{SNe}$ \\
\hline$\chi_{\min }^{2}$ & 9.40 & 544.12 & 554.39 \\
$\Omega_{2,0}$ & $0.277_{-0.097}^{+0.098}$ & $0.288_{-0.105}^{+0.108}$ & $0.281_{-0.071}^{+0.072}$ \\
$n$ & $0.70_{-1.54}^{+1.54}$ & $0.17_{-1.10}^{+1.10}$ & $0.22_{-0.66}^{+0.67}$ \\
$w_{\mathrm{IDM}}$ & $-1.23_{-0.51}^{+0.51}$ & $-1.06_{-0.37}^{+0.37}$ & $-1.07_{-0.23}^{+0.24}$ \\
\hline
\end{tabular}

$H(z)$ data $\left(n \simeq-0.30\right.$ and $w_{\text {IDM }} \simeq-0.90$, which favors the effective quintessence model with a constant EoS for which $w>-1$ ). In Fig. 5, we show the constraint results from SNe Ia. By minimizing the corresponding $\chi^{2}$, we find that the best-fit model values are $\Omega_{2,0}=0.288_{-0.105}^{+0.108}$ and $n=0.17_{-1.10}^{+1.10}\left(w_{\text {IDM }}=-1.06_{-0.37}^{+0.37}\right)$. For the combined data $H(z)+\mathrm{SNe}$ shown in Fig. 6, we find the best-fit values are $\Omega_{2,0}=0.281_{-0.071}^{+0.072}$ and $n=0.22_{-0.66}^{+0.67}$ $\left(w_{\text {IDM }}=-1.07_{-0.23}^{+0.24}\right)$. Obviously, all of the above constraints are consistent with the concordance $\Lambda \mathrm{CDM}$ model and appear to be most consistent with the effective phantom model and a constant EoS for which $w<-1$.

\section{Conclusions}

We have investigated the interacting dark matter (IDM) scenario mimicking either the $\Lambda \mathrm{CDM}$ model or the $w \mathrm{CDM}$ model, which can create the cosmic acceleration without dark energy (Basilakos \& Plionis 2009). In our work, the scale of the effective annihilation term $\kappa C_{1}$ and therefore the mass of DE particles $M_{x}$ are constrained with different newly revised observational data including $H(z)$ and Union2 SNe Ia data. When mimicking a $\Lambda$ cosmology and using three different data combinations of $H(z), \mathrm{SNe} \mathrm{Ia}$, and $\mathrm{SNe} \mathrm{Ia}+H(z)$, we have found that $\kappa C_{1}$ is quite small, which is consistent with the previous results in Basilakos \& Plionis (2009), which used fewer observational $H(z)$ data. Meanwhile, for the combined data sets, we obtain a more stringent upper limit to the effective annihilation term with $\log \left(\kappa C_{1} \cdot \mathrm{Gyr}\right) \approx-3.4$. By relating the range of values of $\kappa C_{1}$ to the mass of the DM particle, we have inferred an apparent lower limit of $M_{x} \approx 10^{-8.6} \mathrm{Gev}$. Furthermore, when mimicking $\omega \mathrm{CDM}$ model and assuming that the particle creation term dominates $(\kappa=0)$, we obtained the effective equation of state of IDM is consistent with the concordance $\Lambda \mathrm{CDM}$ model and appears to be consistent with the effective phantom model with a constant EoS for which $w<-1$.

To sum up, we conclude that the interacting dark matter (IDM) model may provide a practical alternative to dark energy in explaining the present cosmic acceleration. The hope of proving this conclusion should be pinned on analyses of future 
observational data such as high redshift SNe Ia data from SNAP (Albrecht et al. 2006), more precise CMB data from the ESA Planck satellite (Balbi 2007), and complementary data, such as the X-ray gas mass fraction in clusters (Allen et al. 2004, 2008; Ettori et al. 2009), gravitational lensing data (Zhu 1998; Sereno 2002), as well as gamma-ray bursts (GRBs) at high redshift (Schaefer 2007; Liang et al. 2008; Basilakos \& Perivolaropoulos 2008; Liang \& Zhang 2008; Wang \& Liang 2010; Gao et al. 2010; Liang et al. 2011).

Acknowledgements. We thank Yun Chen, Hao Wang, Yan Dai, Chunhua Mao, Fang Huang, Yu Pan, Jing Ming, Kai Liao and Dr. Yi Zhang for discussions. This work was supported by the National Science Foundation of China under the Distinguished Young Scholar Grant 10825313, the Key Project Grants 10533010, and by the Ministry of Science and Technology national basic science Program (Project 973) under grant No. 2007CB815401.

\section{References}

Abraham, R. G., Glazebrook, K., McCarthy, P. J., et al. 2004, ApJ, 127, 2455

Albrecht, A., et al. 2006, Report of the Dark Energy Task Force [arXiv:0609591]

Allen, S. W., Schmidt, R. W., Ebeling, H., Fabian, A. C., \& van Speybroeck, L. 2004, MNRAS, 353, 457

Allen, S. W., Rapetti, D. A., Schmidt, R. W., et al. 2008, MNRAS, 383, 879

Amanullah, R., Lidman, C., Rubin, D., et al. (Supernova Cosmology Project Collaboration) 2010, ApJ, 716, 712

Astier, P., Guy, J., Regnault, N., et al. 2006, A\&A, 447, 31

Balakin, A. B., Pavon, D., Schwarz, D. J., \& Zimdahl, W. 2003, N. J. Phys, 5, 85 Balbi, A. 2007, New A. R., 51, 281

Basilakos, S., \& Lima, J. A. S. 2010, Phys. Rev. D, 82, 023504

Basilakos, S., \& Perivolaropoulos, L. 2008, MNRAS, 391, 411

Basilakos, S., \& Plionis, M. 2009, A\&A, 507, 47

Bengochea, G. R., \& Ferraro, R. 2009, Phys. Rev. D, 79, 124019

Bento, M. C., Bertolami, O., \& Sen, A. A. 2002, Phys. Rev. D, 66, 043507

Cao, S., \& Liang, N. 2010 [arXiv: 1012 . 4879]

Caldwell, R. 2002, PLB, 545, 23

Caldwell, R., Dave, R., \& Steinhardt, P. J. 1998, PRL, 80, 1582

Capozziello, S., \& Fang, L. Z. 2002, Internat. J. Mod. Phys. D, 11, 483

Carroll, S. M., Duvvuri, V., Trodden, M., \& Turner, M. S. 2004, Phys. Rev. D, 70,043528

Chen, G., Gott, J. R., III, \& Ratra, B. 2003, Publ. Astron. Soc. Pac., 115, 1269

Chen, Y., Zhu, Z.-H., Alcaniz, J. S., \& Gong, Y. G. 2010, ApJ, 711, 439

Cirelli, M., Iocco, F., \& Panci, P. 2009, JCAP, 10, 9

Cohen, A., Kaplan, D., \& Nelson, A. 1999, PRL, 82, 4971

Davis, T. M., Mörtsell, E., Sollerman, J., et al. 2007, ApJ, 666, 716

Dvali, G., Gabadadze, G., \& Porrati, M. 2000, PLB, 485, 208

Di Pietro, E., \& Claeskens, J. F. 2003, MNRAS, 341, 1299

Ettori, S., Morandi, A., Tozzi, P., et al. 2009, A\&A, 501, 61

Farrar, G. R., \& Peebles, P. J. E. 2004, ApJ, 604, 1

Feng, B., Wang, X., \& Zhang, X. 2005, PLB, 607, 35

Freedman, W. L., Madore, B. F., Gibson, B. K., et al. 2001, ApJ, 553, 47

Freese, K., \& Lewis, M. 2002, PLB, 540, 1

Gao, H., Liang, N., \& Zhu, Z.-H. 2010 [arXiv: 1003 . 5755]

Gaztañaga, E., Cabré, A., \& Hui, L. 2009, MNRAS, 399, 1663

Gong, Y. G., Cai, R. G., Chen, Y., \& Zhu, Z.-H. 2010, JCAP, 01, 019

Gott, J. R., III, Vogeley, M. S., Podariu, S., \& Ratra, B. 2001, ApJ, 549, 1

Gubser, S. S., \& Peebles, P. J. E. 2004, Phys. Rev. D, 70, 123510

Guo, Z.-K., Piao, Y.-S., Zhang, X., \& Zhang, Y. Z. 2005, PLB, 608, 177

Hicken, M., Wood-Vasey, W. M., Blondin, S., et al. 2009, ApJ, 700, 1097

Jassal, H. K., Bagla, J. S., \& Padmanabhan, T. 2010, MNRAS, 405, 2639

Jimenez, R., Verde, L., Treu, T., \& Stern, D. 2003, ApJ, 593, 622

Kamenshchik, A., Moschella, U., \& Pasquier, V. 2001, PLB, 511, 265

Kolb, E. W., \& Turner, M. S. 1990, The Early Universe (Addison-Wesley Publishing)
Kowalski, M., Rubin, D., Aldering, G., et al. 2008, ApJ, 686, 749

Kurek, A., \& Szydlowski, M. 2008, ApJ, 675, 1

Lazkoz, R., \& Majerotto, E. 2007, JCAP, 0707, 015

Lewis, A., \& Bridle, S. 2002, Phys. Rev. D, 66, 103511

Li, M. 2004, PLB, 603, 1

Liang, N., \& Zhang, S. N. 2008, AIP Conf. Proc., 1065, 367

Liang, N., \& Zhu, Z.-H. 2010, Res. Astron. Astrophys., in press [arXiv: 1010.2681]

Liang, N., Xiao, W. K., Liu, Y., \& Zhang, S. N. 2008, ApJ, 685, 354

Liang, N., Gao, C. J., \& Zhang, S. N. 2009, Chin. Phys. Lett., 26, 069501

Liang, N., Wu, P., \& Zhang, S. N. 2010a, Phys. Rev. D, 81, 083518

Liang, N., Wu, P., \& Zhu, Z.-H. 2010b [arXiv: 1006. 1105]

Liang, N., Xu, L., \& Zhu, Z.-H. 2011, A\&A, 527, A11

Lima, J. A. S., Silva, F. E., \& Santos, R. C. 2008, Class and Quantum Gravity, 25,205006

Ma, C., \& Zhang, T. J. 2011, ApJ, 730, 74

Nolan, L. A., Dunlop, J. S., Jimenez, R., \& Heavens, A. F. 2003a, MNRAS, 341, 464

Nolan, P. L., Tompkins, W. F., Grenier, I. A., \& Michelson, P. F. 2003b, ApJ, 597, 615

Peebles, P. J. E., \& Ratra, B. 1988a, ApJ, 325, L17

Peebles, P. J. E., \& Ratra, B. 1988b, Phys. Rev. D, 37, 3406

Perlmutter, S., Aldering, G., Goldhaber, G., et al. 1999, ApJ, 517, 565

Ratra, B., \& Peebles, P. J. E. 1988, Phys. Rev. D, 37, 3406

Riess, A. G., Filippenko, A. V., Challis, P., et al. 1998, AJ, 116, 1009

Riess, A. G., Strolger, L.-G., Tonry, J., et al. (Supernova Search Team Collaboration) 2004, ApJ, 607, 665

Riess, A. G., Strolger, L.-G., Casertano, S., et al. 2007, ApJ, 659, 98

Riess, A. G., Macri, L., Li, W., et al. 2009, ApJS, 183, 109

Sahani, T. D., Raychaudhury, S., Sahni, V., \& Starobinsky, A. A. 2000, PRL, 85, 1162

Samushia, L., \& Ratra, B. 2006, ApJ, 650, L5

Schaefer, B. E. 2007, ApJ, 660, 16

Sen, A. A., \& Scherrer, R. J. 2008, PLB, 659, 457

Sereno, M. 2002, A\&A, 393, 757

Simon, J., Verde, L., \& Jimenez, R. 2005, Phys. Rev. D, 71, 123001

Stern, D., Jimenez, R., Verde, L., Kamionkowski, M., \& Stanford, S. A. 2010, JCAP, 02, 008

Tammann, G. A., Sandage, A., \& Reindl, B. 2008, A\&ARv, 15, 289

Treu, T., Stiavelli, M., Møller, P., Casertano, S., \& Bertin, G. 2001, MNRAS, 326, 221

Treu, T., Stiavelli, M., Casertano, S., Møller, P., \& Bertin, G. 2002, ApJ, 564, L13

Wan, H. Y., Yi, Z. L., Zhang, T. J., \& Zhou, J. 2007, PLB, 651, 352

Wang, T. S., \& Liang, N. 2010, ScChG, 53, 1720

Wang, S., Li, X. D., \& Li, M. 2011, Phys. Rev. D, 83, 023010

Wei, H. 2010, JCAP, 08, 020

Wei, H., \& Zhang, S. N. 2007, PLB, 644, 7

Wilson, K. M., Chen, G., \& Ratra, B. 2006, Modern Phys. Lett. A, 21, 2197

Wood-Vasey, W. M., Miknaitis, G., Stubbs, C. W., et al. 2007, ApJ, 666, 694

Wu, P. X., \& Yu, H. W. 2007a, PLB, 644, 16

Wu, P. X., \& Yu, H. W. 2007b, JCAP, 0703, 015

Wu, P. X., \& Yu, H. W. 2010, PLB, 693, 415

Xu, L. X., \& Wang, Y. T. 2010a, Phys. Rev. D, 82, 043503

Xu, L. X., \& Wang, Y. T. 2010b, JCAP, 11, 014

Xu, L. X., \& Wang, Y. T. 2010c [arXiv: 1009.0963]

Xu, L. X., Zhang, C. W., Chang, B. R., \& Liu, H. Y. 2008, Modern Phys. Lett. A, 23, 1939

Yi, Z. L., \& Zhang, T. J. 2007, Modern Phys. Lett. A, 22, 41

Zhai, Z. X., Wan, H. Y., \& Zhang, T. J. 2010, PLB, 689, 8

Zhang, H. S., \& Zhu, Z.-H. 2008, JCAP, 0803, 007

Zhang, T. J., Ma, C., \& Lan, T. 2010, Adv. Astron., 184284

Zimdahl, W., Schwarz, D. J., Balakin, A. B., \& Pavon, D. 2001, Phys. Rev. D, 64,3501

Zhu, Z.-H. 1998, A\&A, 338, 777

Zhu, Z.-H., \& Alcaniz, J. S. 2005, ApJ, 620, 7

Zhu, Z.-H., \& Fujimoto, M.-K. 2002, ApJ, 581, 1 